\title{
Morphology of planetary nebulae with binary cores
}

\section{The effect of gravitational focusing by the companion to the mass-losing star}

\author{
A. J. Gawryszczak ${ }^{1}$, J. Mikołajewska ${ }^{1}$, and M. Różyczka ${ }^{1}$
}

Nicolaus Copernicus Astronomical Center, Bartycka 18, Warsaw 00-716, Poland

Received 30 July 2001 / Accepted 13 December 2001

\begin{abstract}
We present hydrodynamical models of planetary nebulae with detached binary cores. The models are obtained according to the interacting winds scenario. Slow AGB wind models are produced by means of 3-D SPH simulations. The SPH results provide initial conditions for the evolution of a fast and rarefied wind injected into the AGB wind. In close binaries the density distribution of the slow wind is significantly modified by the gravity of the secondary, resulting in an enhanced density region close to the orbital plane of the system, and low density regions elongated perpendicularly to the orbital plane (we refer to those effects as gravitational focusing). The fast wind propagating through such a medium naturally develops a bipolar structure. In wider binaries the effect of gravitational focusing is weaker, and elliptical nebulae are produced instead of bipolar ones. In binaries wider than $\sim 10 \mathrm{AU}$ the effect becomes unimportant, and the resulting nebulae are almost entirely spherical apart from local corrugations caused by hydrodynamical instabilities. Thus, gravitational focusing alone is capable of generating a broad range of morphologies (from nearly spherical to strongly bipolar). The results are discussed in relation to nebulae associated with symbiotic stars.
\end{abstract}

Key words. planetary nebulae: general - stars: winds, outflows - stars: binaries: symbiotic - hydrodynamics

\section{Introduction}

According to the widely accepted two-wind scenario, a planetary nebula (PN) is formed when a high-speed, lowdensity wind emitted by the central star interacts with a slow, high-density wind emitted by the same star in its AGB evolutionary phase (see e.g. García-Segura et al. 1999 and references therein). Elliptical or bipolar nebulae are easily produced when the density distribution of the slow wind has a maximum in a well-defined plane (see e.g. Matt et al. 2000; Dwarkadas \& Balick 1998; Mellema 1997; Mellema \& Frank 1995; Mellema 1995; Soker 1998, and references therein). Among various mechanisms that may produce such a distribution, gravitational deflection of the wind by a companion to the mass-losing star is particularly simple and appealing. However, despite its simplicity and attractiveness its influence on nebular morphology has not been studied in detail.

From the observational point of view, the question of whether close companions strongly influence or even cause the ejection of asymmetric planetary nebulae (PNe) is open because definitive evidence is still missing. The very important observational constraint that bipolar $\mathrm{PNe}$ are produced by more massive progenitors, $>1.3 M_{\odot}$, than

Send offprint requests to: A. J. Gawryszczak, e-mail: gawrysz@camk.edu.pl the other morphological types, does not help to discriminate between single and binary star models, as both provide an explanation for that (Corradi 2000, and references therein).

While all planetary nebulae with short-period binary nuclei (periods up to a few days) have aspherical structures, including bipolar, elliptical, or ring morphologies, one cannot claim that all aspherical PNe have been ejected from such binaries (Bond (2000) and references therein). The situation looks more promising for systems with longer orbital periods, like symbiotic stars (interacting binaries composed of an evolved giant primary and a hot white dwarf secondary surrounded by an ionized nebula). Most of resolved nebulae associated with symbiotic systems are evidently aspherical, and about $50 \%$ of them have a bipolar/ring morphology (e.g. Corradi et al. 1999). Among all PNe only about $15 \%$ are bipolar, while about $20 \%$ are spherically symmetric. This statistics alone is a clear indication that interactions in binary systems favor formation of bipolar and aspherical nebulae (Corradi 2000).

The main effect of the secondary on the AGB wind from the primary is gravitational deflection of the latter toward the orbital plane (hereafter referred to as gravitational focusing). As a result, an enhanced density region is created at the orbital plane together with low density 
regions elongated perpendicularly to the orbital plane. A fast wind interacting with such a medium naturally produces an aspherical nebula. The aim of the present paper is to establish the range of resulting nebular morphologies.

We employ SPH (Smoothed Particle Hydrodynamics) techniques to obtain 3-D models of AGB winds from binary systems on scales of up to several tens of $\mathrm{AU}$. The SPH models are subsequently used to generate initial and boundary conditions for grid-based simulations of fast wind evolution on much larger scales. Our research is limited to the consequences of gravitational focusing alone. Any additional effects, resulting e.g. from magnetic fields or circum-secondary disks with associated jets, are not included. While they are undoubtedly needed to account for more exotic morphologies (e.g. point symmetry), their physical foundations are as yet rather weak. Contrary to that, the physics of gravitational focusing is well understood, and related simulations do not have to rest on more or less arbitrary assumptions. We also neglect ionization, as it is responsible mainly for small-scale structures, whereas our concern here is global morphology of the nebula.

The paper is organized as follows: in Sect. 2 numerical techniques and details of the physical setup of the models are discussed. The models are presented in Sect. 3, which also contains the discussion of our results in relation to symbiotic systems. Section 4 provides a brief summary of our work.

\section{Methods and assumptions}

As mentioned in Sect. 1, our models are constructed in a two-step procedure. First, we obtain three-dimensional stationary density distributions of dense winds from an AGB star in a binary system. For that purpose, the SPH technique is used (see e.g. Hernquist \& Katz 1989). Our implementation of the SPH technique is based on variable smoothing length with spline kernel introduced by Monaghan \& Lattanzio (1985). The number of neighbouring particles is set to 40 . Similar research was already performed by Mastrodemos \& Morris (1998, 1999). In many aspects the work they report is superior to ours: a more sophisticated physics is employed, and the numerical resolution is better. However, as far as the stationary density distribution of the AGB wind is concerned, our results are entirely compatible with theirs.

The SPH models provide realistic initial conditions for the second step, in which the evolution of fast winds is followed by means of grid-based simulations performed with the ZEUS code (Stone \& Norman 1992). This step is crucial for our work, as in most PNe simulations performed to date the density distributions of AGB winds have been more or less arbitrary, see e.g. Dwarkadas \& Balick (1998). Our procedure is free from such arbitrary assumptions, and our model PNe provide a comprehensive illustration of a physically plausible ("waterproof") mechanism responsible for nebular asphericities.

\subsection{SPH models}

For both SPH and ZEUS calculations an inertial coordinate frame is used, with the origin at the mass center of the system (Fig. 1). The AGB star is assumed to rotate synchronously, but it does not fill its Roche lobe, so that the mass flow through the inner Lagrangian point can be neglected. The AGB wind is represented by a stream of particles launched at a constant rate from points distributed randomly on the surface of the primary. For practical reasons (CPU time), the total number of particles in the computational domain must be limited. Thus, whenever the pre-set limit of $8 \div 9 \times 10^{4}$ particles is exceeded, those in excess of the limit, and most distant from the wind source, are removed from the domain. We have found that the distance beyond which removals occur $\left(r_{\text {rem }}\right)$ stabilizes within several orbital periods of the binary $\left(P_{\text {orb }}\right)$, and an almost stationary rotating density distribution is obtained. With the above limit imposed on the number of particles, in most cases $r_{\text {rem }}$ does not exceed $50 \mathrm{AU}$. As the details of the flow in the vicinity of the secondary (e.g. accretion disk) are out of scope of the present paper, all particles within $0.1 \mathrm{AU}$ from the secondary are also removed, provided that their velocities are directed toward the secondary (otherwise, due to large particle velocities, time-step would become prohibitively short).

We assume that the AGB wind is composed of dust and ideal monoatomic gas which are dynamically coupled (i.e. they move at the same velocity). The details of wind acceleration process are not followed. Finally, we assume that the gravity of the AGB star is balanced by radiation pressure, so that it does not appear explicitly in our equations. If the mass-losing star were single, the only accelerating force acting on the wind would be due to pressure gradients. In a binary, the wind is additionally subject to the gravity of the companion. Obviously, such an approximation is rather crude. However, our winds reach terminal velocities comparable to those given by Winters et al. (2000) for mass loss rates $\sim 10^{-6} M_{\odot} \mathrm{yr}^{-1}$ which, in turn, are obtained when radiation pressure nearly balances the gravity of the wind source. Radiation pressure from the secondary, and self-gravity of the wind are neglected. We also neglect radiative heating and cooling, and employ a polytropic equation of state $p=\kappa \rho^{\gamma}$, with $\gamma=1.66$.

The initial temperature of wind particles is $3000 \mathrm{~K}$, and in most cases their initial velocity is set to $1 \mathrm{~km} \mathrm{~s}^{-1}$. Both parameters do not depend on the location of the particle on the surface of the star. The particles that have left the star are accelerated by wind pressure gradient and secondary's gravity. The wind generation procedure has been tested on a freely expanding wind from a single star. In Fig. 2 numerical results of the test are compared to solutions of equations

$$
\begin{aligned}
v(r) & =\frac{\alpha}{r^{2} \rho(r)} \\
\frac{\mathrm{d} \rho}{\mathrm{d} r} & =\frac{\rho}{r} \frac{2}{\kappa \alpha^{-2} \gamma r^{4} \rho^{\gamma+1}-1}
\end{aligned}
$$




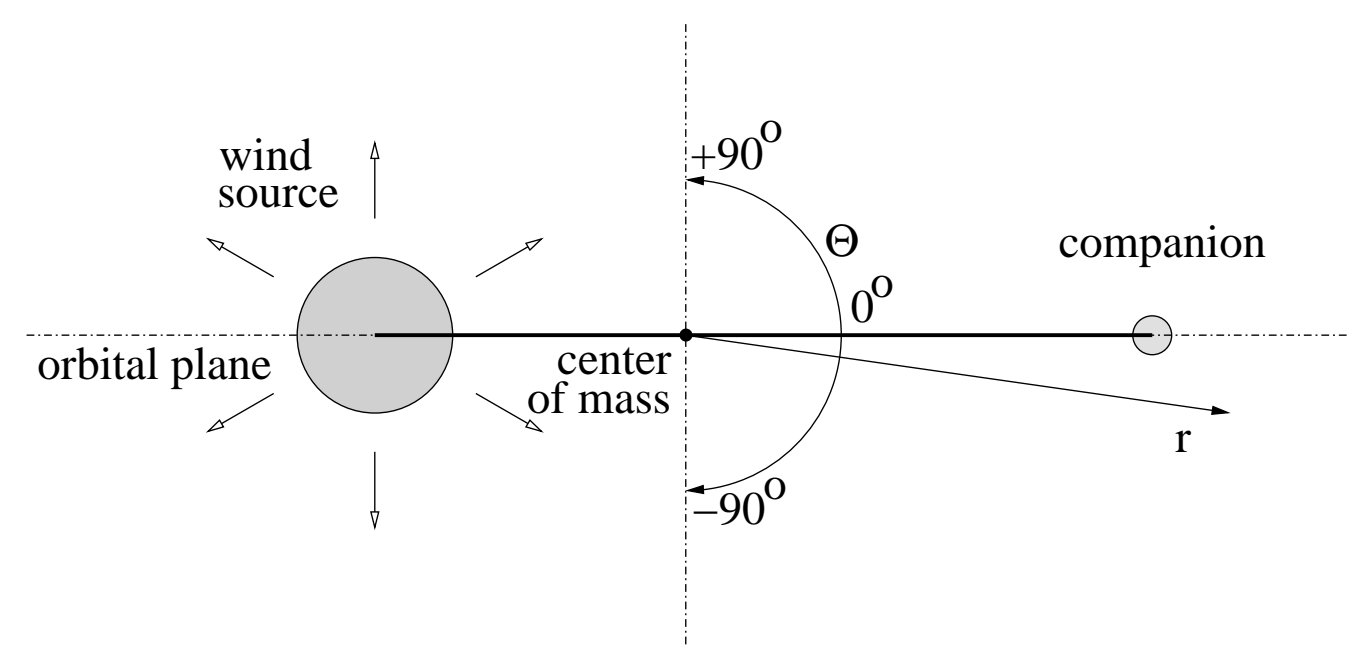

Fig. 1. Schematic side-view of the central part of the computational domain. The third coordinate is the azimuthal angle $\phi$ defined in the orbital plane.
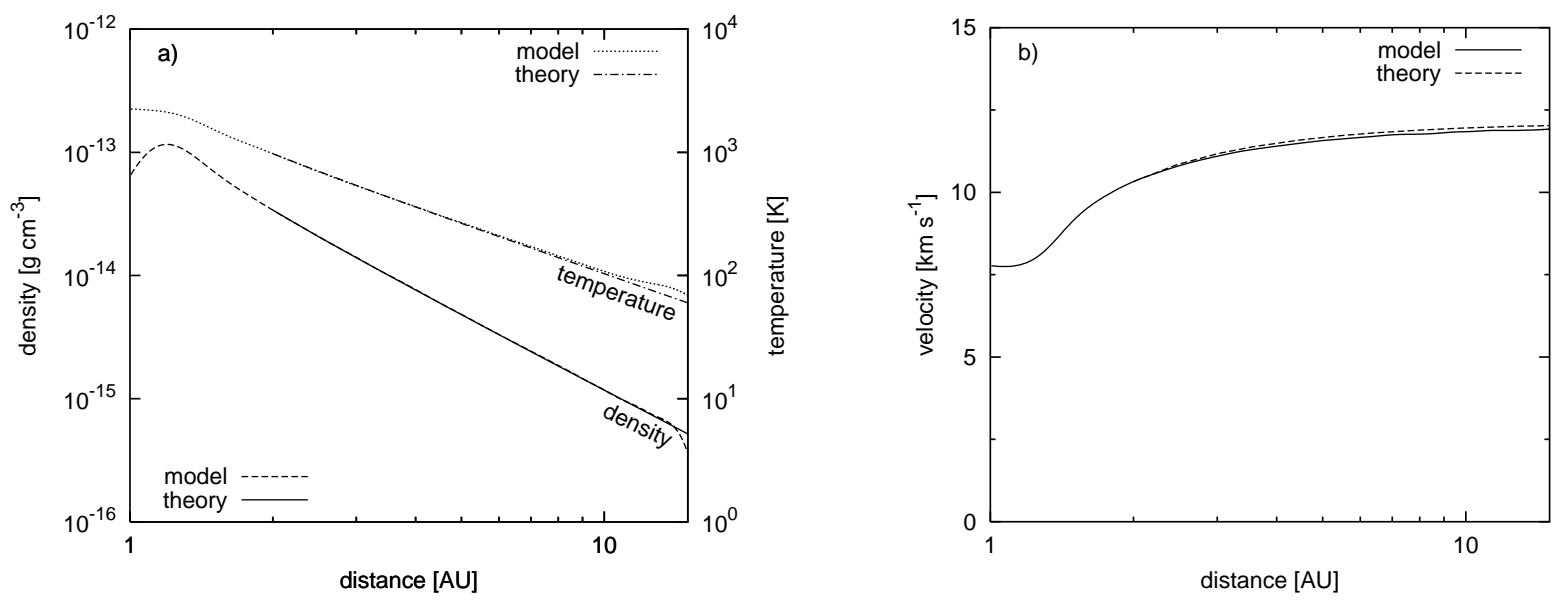

Fig. 2. Freely expanding, spherically-symmetric wind. SPH results averaged over the entire solid angle (label: model) are compared to the analytical solution (label: theory). Initial values of $\rho, v$ and $T$ for the analytical solution are taken from the $\mathrm{SPH}$ model at $r=2 \mathrm{AU}$. SPH particles are injected at $r_{0}=1 \mathrm{AU}$ with $v_{0}=8.9 \mathrm{~km} \mathrm{~s}^{-1}$ and temperature $T_{0}=3000 \mathrm{~K}$. In the left panel theoretical and SPH density curves practically coincide. Note that the effective temperature of the wind at $1 \mathrm{AU}$ (estimated from the extrapolation of the analytical curve) is different from the assumed one. This is because we do not have any ghost particles at $r<1 \mathrm{AU}$.

where $\alpha=\dot{M} / 4 \pi$ (derivation of Eq. (2) can be found in Appendix A).

There are good reasons to believe that winds from AGB stars are intrinsically aspherical (see e.g. Frankowski \& Tylenda 2001; Dorfi \& Höfner 1996; Reimers et al. 2000, and references therein). This effect may arise from internal processes taking place in the star and/or the presence of its companion. Since the theory is not yet advanced enough for precise quantitative predictions, in our models the intrinsic asymmetry is taken into account according to a simple prescription

$F\left(\Theta^{\prime}, \phi^{\prime}\right) \propto 1+b\left(\left(1+q\left(3 \cos ^{2} \phi^{\prime}+1\right)\right) \cos ^{2} \Theta^{\prime}-q\right)$

where $F$ is the mass flux per unit solid angle, $q=M_{2} / M_{1}$ is the secondary-to-primary mass ratio, $b$ is a scaling parameter, and $\left(\Theta^{\prime}, \phi^{\prime}\right)$ are defined on primary's surface (orbital plane is located at $\Theta^{\prime}=0^{\circ}$, and $\phi^{\prime}$ is measured from the axis pointing to the companion). The above equation is derived from Taylor expansion of more general formulae given by Frankowski \& Tylenda (2001) (their Eqs. (14), (18) and (26); note that their effective gravity refers to deeper layers of primary's atmosphere, and, unlike ours, it cannot be equal to 0 ).

We define a convenient measure of intrinsic asphericity, $\mathcal{A}$, as the ratio of mean mass flux per unit solid angle at the orbital plane $\left(\Theta=0^{\circ}\right)$ to the mass flux per unit solid angle in the direction perpendicular to the orbital plane,

$\mathcal{A}=\frac{\frac{1}{2 \pi} \int_{0}^{2 \pi} F\left(0^{\circ}, \phi^{\prime}\right) \mathrm{d} \phi^{\prime}}{F\left(90^{\circ}, \phi^{\prime}\right)}$

so that

$b=\frac{\mathcal{A}-1}{q\left(\mathcal{A}+\frac{3}{2}\right)+1}$. 
According to Frankowski \& Tylenda (2001), $\mathcal{A}$ hardly exceeds 2. Dorfi \& Höfner (1996) and Reimers et al. (2000) discuss single AGB stars with rotation periods corresponding to orbital periods of our binary systems. They easily obtain $\mathcal{A}>2$; however, only for stars with rotational periods shorter than $\sim 20 \mathrm{yr}$.

Our experience shows that AGB winds with $\mathcal{A}<2$ (including an entirely spherical one) are similar enough to yield planetary nebulae of the same shape. On the other hand, strong intrinsic asymmetries $(\mathcal{A} \gg 2)$ would override the effects of gravitational deflection by the secondary which we are mostly interested in. Therefore, in the present paper $\mathcal{A}=2$ is only considered. To account for the intrinsic asymmetry, the locations of particle injection points are drawn from a distribution based in Eq. (3).

In the following, the term "SPH model" will refer to the stationary configuration of an AGB wind obtained from SPH simulations. Apart from density contrasts in $\Theta$, our SPH models display a regular spiral structure in $\phi$, also observed by Mastrodemos \& Morris $(1998,1999)$. The spiral density enhancements are best seen in the $\Theta=0^{\circ}$ cross-sections of the models (Fig. 3). As one might expect, spirals resulting from close binaries are more tightly wound than those from wider ones.

The spirals diffuse due to pressure gradients on a characteristic time scale $t_{\mathrm{d}}=\lambda / c$, where $\lambda$ is the radial distance between neighbouring crests of the spiral wave, and $c$ is the local velocity of sound. A good approximation for $\lambda$ is the product of local wind velocity $v_{\mathrm{w}}$ and $P_{\text {orb }}$. The decay of the spiral design is completed (or, at least, significantly advanced) at $r_{\mathrm{d}}$, where

$r_{\mathrm{d}} \simeq t_{\mathrm{d}} \cdot v_{\mathrm{w}}=P_{\mathrm{orb}} \cdot v_{\mathrm{w}}^{2} / c$.

With $P_{\text {orb }} \sim 20$ years, $v_{\mathrm{w}} \sim 10 \mathrm{~km} \mathrm{~s}^{-1}$ and $c \sim 3 \mathrm{~km} \mathrm{~s}^{-1}$, $r_{\mathrm{d}}$ does not exceed $100 \mathrm{AU}$.

One may observe in Fig. 3 that the amplitude of the spiral indeed decreases with $r$. However, this effect must be viewed with some caution. One should remember that a variable smoothing length causes loss of numerical resolution with increasing $r$. At large distances from the binary, the size of the details we can resolve, $\delta r$, is proportional to the distance from the center of mass, $r$. In the left panel of Fig. $3 \delta r$ increases from 1.5 to $4.5 \mathrm{AU}$ between 10 and $35 \mathrm{AU}$, respectively, and in the left panel - from 7 to $17 \mathrm{AU}$ between 40 and $100 \mathrm{AU}$, respectively. Upon comparing $\delta r$ to the radial wavelength of spirals in Fig. 3 one may infer that numerical diffusion dominates in the left panel at $r \gtrsim 20 \mathrm{AU}$, whereas in the right panel physical effects of pressure gradients can be observed for the whole range of $r$. As the amplitude of spirals decreases at large $R$, the density distribution becomes more axially symmetric. This effect is crucial to our grid-based simulations.

Upon concluding this section let us note that the equations governing the propagation of adiabatic AGB winds can be written in a system of units in which gravitational constant, primary's mass, and $a(1+q)$ are all equal to 1 . Such an operation demonstrates that the only independent parameters of the model are mass ratio $q$, initial wind velocity (expressed in units equal to secondary's orbital velocity), and initial wind temperature measured in virial units of the system. Unfortunately, those simple scaling properties are lost as soon as radiative cooling is included in the physics of the models.

\subsection{Grid-based models}

Our ultimate aim is to obtain models of planetary nebulae resulting from the expansion of a fast, low-density wind in the dense wind emitted by the primary during its AGB phase. To that end, we have to expand the SPH models obtained in Sect. 2.1, whose sizes in most cases do not exceed $50 \mathrm{AU}$. We have found that in all cases the AGB wind reaching the outer boundary of the computational domain is supersonic, with the radial Mach number ranging from $\sim 3$ to $\sim 7$, and with nonradial velocities smaller than a few per cent of the radial velocity. Thus, our models may be extrapolated beyond $r_{\text {rem }}$ analytically with the help of Eqs. (A.4) applied locally (i.e. for every direction separately), using values of $\rho\left(r_{\text {rem }}\right)$ and $v\left(r_{\text {rem }}\right)$ as initial conditions.

Before extrapolation, we apply averaging over a few consecutive time steps in order to reduce numerical noise in low-density regions. Next, based on the decay of nonaxisymmetric features discussed in Sect. 2.1, the models are averaged over $\phi$. Any residual small-amplitude fluctuations in $\Theta$ are additionally smoothed with a low-pass filter. The resulting axisymmetric configurations may be investigated by means of 2 -D simulations at a modest expense of CPU time.

Smoothed SPH models are expanded up to $r=610 \mathrm{AU}$ and interpolated onto a two-dimensional $(r, \Theta)$ grid of the ZEUS code. The grid consists of 600 and 200 points in $r$ and $\Theta$, respectively, with $\Theta$ extending from $-90^{\circ}$ to $+90^{\circ}$. At the start of each simulation the central region $(r<40 \mathrm{AU})$ of the model is filled with an isotropic flow representing the fast, low-density wind, and later on the wind is injected at a constant rate through the inner boundary of the grid (allowing for a finite initial thickness of the free wind region we avoid spurious interactions between reverse shock and inner grid boundary, which, under some circumstances, may lead to unphysical results). Optically thin cooling is applied throughout the evolution, resulting in compression and deformation of shocked layers of gas. Cooling function given by MacDonald \& Bailey (1981) is used.

\section{Results}

Our models are listed in Table 1. In all cases the binary is composed of a $1 M_{\odot}$ AGB star and an $0.6 M_{\odot}$ secondary. The mass loss rates due to AGB and fast winds are $10^{-6}$ and $10^{-8} M_{\odot} \mathrm{yr}^{-1}$, respectively. The velocity of the fast wind is $10^{3} \mathrm{~km} \mathrm{~s}^{-1}$. The models differ in binary separation $a$, size of the primary $r_{0}$ and some AGB wind parameters. The term "resolution" in the last column of Table 1 refers to the mass of a single AGB wind particle, which in 
a)

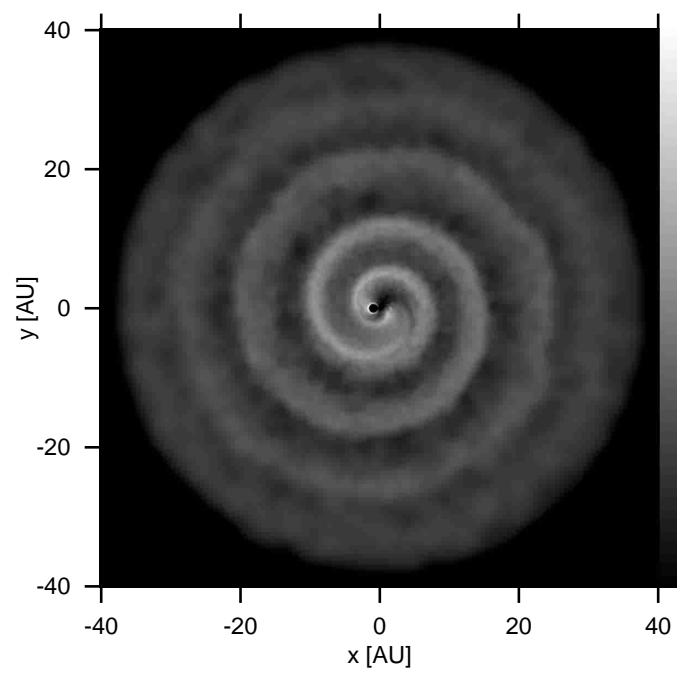

b)

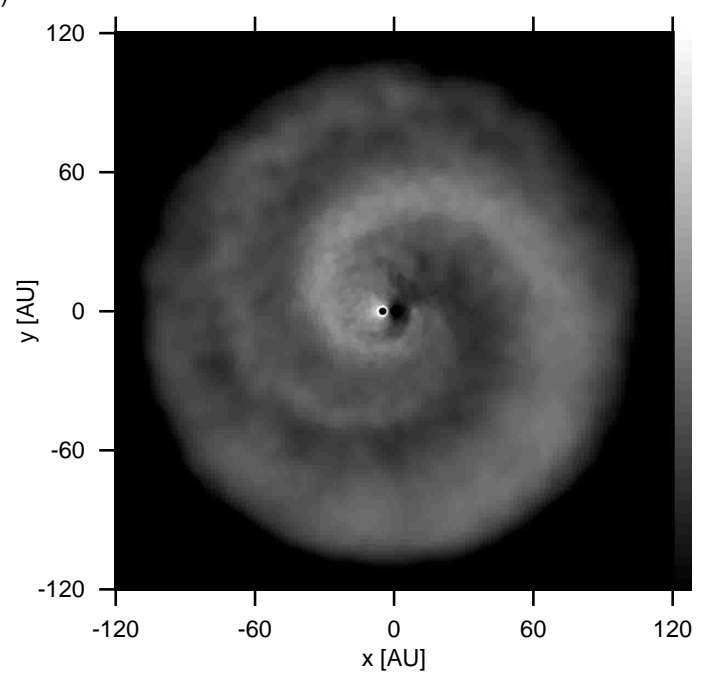

Fig. 3. Quantity $\sigma=\rho \cdot r^{2}$ plotted in the orbital plane of Model C (close binary; a)), and Model I (wide binary; b)). The plot is logarithmic, and the range of $\sigma$ spans 2 orders of magnitude. Black areas at the center are the interior of the primary (inside a bright ring; slightly offset from the center), and the center of the coordinate system, where $\rho$ is finite, but $\sigma$ is close to 0 due to the $r^{2}$ factor. In order to reduce the numerical noise the values of $\sigma$ are averaged over several time-steps. Note that $\sigma=$ const for a uniform, constant velocity wind.

Table 1. Model list.

\begin{tabular}{|c|c|c|c|c|c|c|}
\hline Model & $\begin{array}{l}a \\
{[\mathrm{AU}]}\end{array}$ & $\begin{array}{l}r_{0} \\
{[\mathrm{AU}]}\end{array}$ & $\begin{array}{l}v_{\mathrm{s}} \\
{\left[\mathrm{km} \mathrm{s}^{-1}\right]}\end{array}$ & $\begin{array}{l}v_{\mathrm{a}} \\
{\left[\mathrm{kms}^{-1}\right]}\end{array}$ & $\begin{array}{l}r_{\mathrm{rem}} \\
{[\mathrm{AU}]}\end{array}$ & remarks \\
\hline $\mathrm{A}$ & 2.4 & 0.7 & 15.2 & 9.0 & 14 & higher resolution \\
\hline B & 2.4 & 0.7 & 15.2 & 13.7 & 24 & $v_{0}=12 \mathrm{~km} \mathrm{~s}^{-1}$ \\
\hline $\mathrm{C}$ & 2.4 & 0.7 & 15.2 & 8.7 & 35 & \\
\hline $\mathrm{D}$ & 5.6 & 1.0 & 9.9 & 8.7 & 30 & \\
\hline $\mathrm{E}$ & 8.0 & 1.5 & 8.3 & 9.0 & 42 & \\
\hline $\mathrm{F}$ & 9.6 & 1.5 & 7.6 & 9.0 & 38 & \\
\hline $\mathrm{G}$ & 9.6 & 1.5 & 7.6 & 9.0 & 40 & $\mathcal{A}=0$ \\
\hline $\mathrm{H}$ & 12.6 & 2.0 & 6.6 & 9.3 & 44 & \\
\hline I & 12.6 & 2.0 & 6.6 & 9.3 & 110 & lower resolution \\
\hline
\end{tabular}

Model A is 3 times smaller, and in Model I is 3 times larger than in the remaining ones. The sole purpose of the lowresolution Model $\mathrm{I}$ is to follow the decay of spiral features at large $r$, illustrated in Fig. 3. On the other hand, the high-resolution Model A (otherwise the same as Model C) enables us to follow the details of the flow in low density regions at $\Theta= \pm 90^{\circ}$. For all models orbital velocity of the secondary $v_{\mathrm{s}}$, AGB wind velocity at secondary's orbit $v_{\mathrm{a}}$, and particle removal radius $r_{\text {rem }}$ are also given. A threepercent discrepancy in $v_{\mathrm{a}}$ between Models $\mathrm{A}$ and $\mathrm{C}$ should probably be attributed to the resolution-dependent internal viscosity of the SPH code. In high-resolution Model A $r_{\text {rem }}$ is $14 \mathrm{AU}$, since any larger removal radius would imply a prohibitively large number of particles.

\section{1. $S P H$}

The particles of the AGB wind are injected at a velocity $v_{0}=1 \mathrm{~km} \mathrm{~s}^{-1}$ in all models except $\mathrm{B}$, where $v_{0}$ is raised to $12 \mathrm{~km} \mathrm{~s}^{-1}$. All models except $\mathrm{G}$ are intrinsically asymmetric $\mathrm{AGB}$ winds with $\mathcal{A}=2$. In Model $\mathrm{G}$ the wind is spherically symmetric. For each model, the plots in Fig. 4 show smoothed density and velocity distributions of the AGB wind in the plane perpendicular to the orbit, with density values correspond to $r=610 \mathrm{AU}$. The general effect of the secondary is to deflect the AGB wind toward the orbital plane, i.e. the AGB wind is subject to "gravitational focusing" toward the orbital plane. Focusing efficiency weakens with increasing $a$ (Fig. 4a), so that it is hardly visible in binaries wider than $\sim 10$ AU (models $\mathrm{F}-\mathrm{H}$ ). This is because of increasing ratio of wind velocity at secondary's orbit, $v_{\mathrm{a}}$, to orbital velocity of the secondary, $v_{\mathrm{s}}$. We must stress that in all models presented here the density enhancement in the orbital plane is underestimated due to the absence of radiative cooling (this is because a high-pressure "halo" forms around the secondary, partly preventing the inflow of matter into the orbital plane).

In addition to the density enhancement in the orbital plane, funnel-like density minima at $\Theta=90^{\circ}$ are observed in close binaries (Models A-D). The funnels originate from two factors. First, the wind in a close binary carries appreciable angular momentum, as it is emitted from a source moving at a high orbital velocity. The centrifugal force associated with the specific angular momentum of the wind causes the wind gas to clear the vicinity of the polar axis. Second, gravity of the secondary is not balanced by radiation pressure (unlike in the case of the primary), and as a result the outflow along the axis is retarded. In wider systems this effect is of course weaker. 

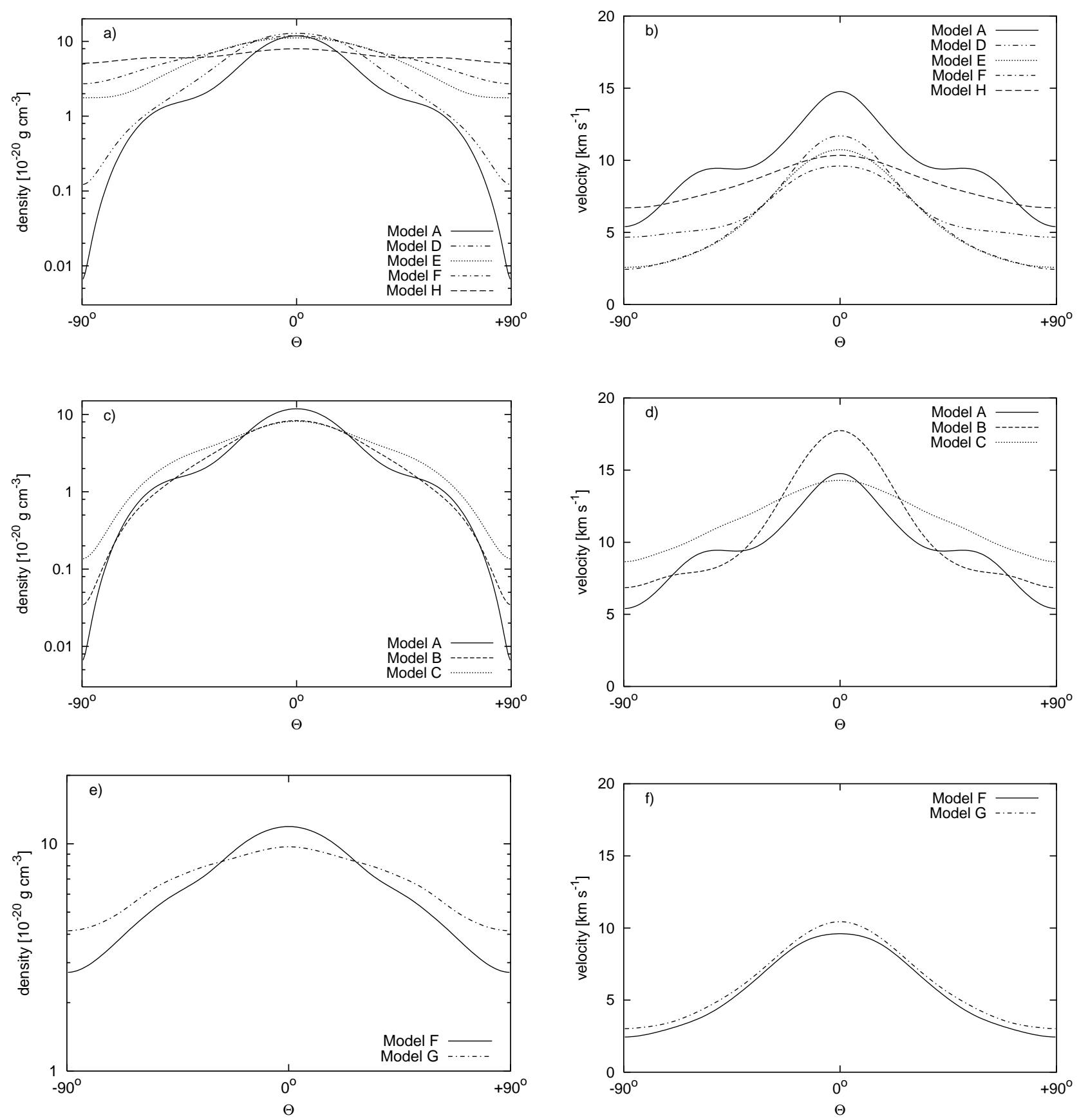

Fig. 4. Smoothed density and velocity distributions of SPH models expanded to 610 AU. a) and b): models with initially slow wind, c) and $\mathbf{d}$ ): close binary models, e) and f): intrinsically spherical model G compared to aspherical model H.

The wave at the wings of density and velocity profiles of Model A (Figs. 4a-d) traces spiral density enhancements discussed in Sect. 2.1. It is barely visible in (or entirely absent from) remaining models because of larger $r_{\text {rem }}$ which results in more advanced decay of spiral features.

The sensitivity of the polar flow to wind parameters and details of numerical procedure is illustrated in Figs. 4c and $\mathrm{d}$. The deepest funnel is obtained in high-resolution Model A. At the standard resolution (Model C) the funnel is much shallower, and velocity profile in $\Theta$ is flattened. This is because larger smoothing length adds more dif- fussivity to the code. Fast wind (Model B) yields polar density minima more clearly pronounced than in Model C because more matter is able to escape along the polar axis from the potential well.

In the absence of the secondary our standard AGB wind would reach an isotropic terminal velocity of $14.4 \mathrm{~km} \mathrm{~s}^{-1}$ for Model B, and $9.1 \pm 0.2 \mathrm{~km} \mathrm{~s}^{-1}$ for all other models. In a binary, the wind is accelerated in the orbital plane due to angular momentum transfer (see Figs. 4b, d, and $\mathrm{f}$ ). The velocity excess at $\Theta=0^{\circ}$, defined as the difference between wind velocity from a single star and effective 
velocity, amounts to $\sim 30 \%$ in close binaries (Models $\mathrm{A}$, $\mathrm{B}, \mathrm{C})$, and is nearly absent in the widest Models $\mathrm{F}$ and $\mathrm{G}$.

In Figs. 4e and $\mathrm{f}$ intrinsically asymmetric Model $\mathrm{F}$ and intrinsically isotropic Model G are compared. The discrepancy between their final density distributions reaches $\sim 30 \%$ at $\Theta= \pm 90^{\circ}$, and only $\sim 10 \%$ at $\Theta=0^{\circ}$ whereas the velocity distributions are nearly identical. In both models the wind is slowed down in polar directions by secondary's gravity.

\subsection{ZEUS}

Models of planetary nebulae resulting from interactions between fast wind and AGB wind are displayed in Figs. 5, 6. Models $\mathrm{C}$ and $\mathrm{D}$, whose density distributions are nearly identical for $|\Theta| \gtrsim 70^{\circ}$ (Figs. 4a, c), produce nebulae that are hardly distinguishable, and only one of them is shown. Nebulae obtained from SPH models G and H are also similar; here in both cases the shape of the nebula is nearly spherical.

The morphology of the nebula depends mainly on the ratio $\epsilon=\rho\left(\Theta=0^{\circ}\right) / \rho\left(\Theta= \pm 90^{\circ}\right)$ obtained in the SPH model. For $\epsilon \gtrsim 10$ (models with deep density minima at $\Theta= \pm 90^{\circ}$ ) bipolar nebulae are produced, extending perpendicularly to the orbital plane. The width of bipolar lobes is determined by the width of polar funnels (which, in turn, is correlated with $\epsilon$ ). Elliptical or spherical nebulae develop for $\epsilon \lesssim 5$ (i.e. in models with no polar funnels). The transition between those two morphological cases is achieved in Model $\mathrm{E}$, in which $\epsilon \simeq 6$.

In the following, the main morphological features of the models are discussed for cases D and F, which are representative for bipolar and elliptical nebulae, respectively. In Fig. 5 the central sphere of each model is the freely expanding fast wind, with density proportional to $r^{-2}$. A sharp increase in density at the boundary of the free wind marks the location of the reverse shock front. In Model D the reverse shock is surrounded by a rather narrow subsonic region, from which the gas escapes along the symmetry axis. The flow along the axis accelerates at the expense of internal energy of the gas to finally enter secondary supersonic regions above and below the orbital plane. Each of them is bordered by a shock pattern composed of three waves, again marked by sharp density jumps (better seen in Fig. 6). The nearly spherical shock at the axis is the strongest of the three, and the gas that has crossed it expands nearly radially. The nearly cylindrical shock at $\sim 100$ AU from the axis is strongly oblique, forcing the wind to flow almost parallel to the axis at a supersonic speed. The third shock results from collision between the post-cylindrical shock flow and the post-spherical shock flow. Secondary supersonic regions and associated shock patterns are entirely absent from Model F, where the velocity of the wind remains subsonic everywhere beyond the reverse shock.

The shocked fast wind is so hot and rarefied that its radiative cooling time is longer than the time it needs to flow through the computational domain. As a result no effects of cooling are observed in it. However, cooling is effective in the much denser shocked AGB wind. A shell of shocked AGB gas, visible as a narrow stripe of enhanced density, envelopes the whole region filled with shocked fast wind. In dense regions of Model D $\left(|\Theta|<50^{\circ}\right)$ the shell is strongly cooled and compressed to grid resolution limit (Fig. 6). Cooling and compression is much less advanced in polar funnels $\left(|\Theta|>70^{\circ}\right)$, where the velocity of the shock sweeping through the AGB wind is high enough (and the AGB wind density is low enough) for cooling time to exceed the age of the nebula. Such effects are not observed in Model F, in which shock velocity variations are smaller due to a more uniform AGB wind density distribution. Strong corrugations of the shell result from hydrodynamical instabilities (Walder \& Folini 1998 and references therein) which, due to insufficient resolution, are only qualitatively represented in our models.

Among close binary models, Model $\mathrm{C}$ displays the same morphological features as Model B. Model A, however, develops a much more complicated shock pattern in the secondary supersonic region, resembling that of an underexpanded jet (three sets of crossing shocks can be seen). We expect this jet to propagate further away from the binary, although its collimation efficiency may decrease in time.

\subsection{Comparison with symbiotic stars}

In symbiotic binaries the cool giant primary is the source of the slow wind, whereas the hot dwarf companion is responsible for shaping the slow wind and it is also the source of the fast wind. The situation is thus somewhat different from our model binary systems where both winds originate from the primary component. However, the offset between the centers of the two winds in symbiotic binary systems is negligible with respect to the size of associated nebulae. Another objection against direct application of our models to symbiotic systems might refer to variability of fast winds from white dwarfs, which are known to undergo periods of low and high activity. However, the final geometry of the slow wind is achieved after just a few orbital cycles, whereas typical duration of low activity phase is measured in hundreds of years.

The cm-submm radio and IR observations indicate typical values of $\dot{M}_{\text {giant }} / v_{\infty}$ of the order of a few $10^{-8} M_{\odot} \mathrm{yr}^{-1} / \mathrm{km} \mathrm{s}^{-1}$ (e.g. Mikołajewska et al. 2001), near to those characterizing our models ${ }^{1}$. The fast wind velocity is usually in the range $\gtrsim 200 \div 1000 \mathrm{kms}^{-1}$, and estimated mass loss rates range from $\sim 10^{-5}$ to $\sim 10^{-9} M_{\odot} \mathrm{yr}^{-1}$ (Mikołajewska 2000) - which is again

\footnotetext{
1 These estimates are based on the assumption of a spherically symmetric wind from the symbiotic giant. Our calculations show significantly enhanced density region close to the orbital plane and low density regions elongated in polar directions. If this is the case in real systems, then the values quoted above are most probably overestimated.
} 


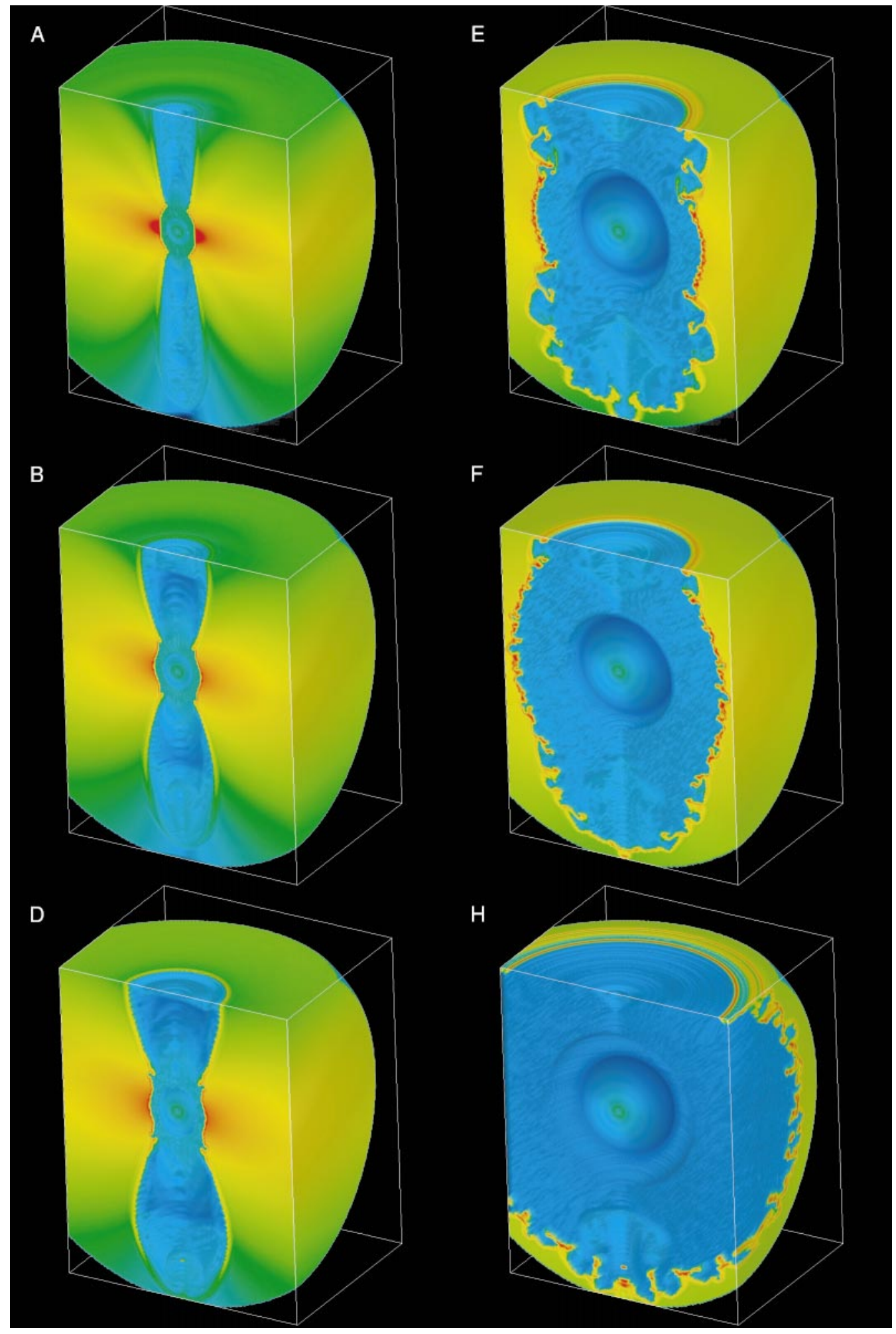

Fig. 5. Volume rendering of final density distributions obtained in models A, B, D, E, F, and H Snapshots are taken at $t=3.80,6.33,12.0,47.5,57.0,79.2$ years, respectively. The inner and outer radius of the spherical computational domain is 10 and $610 \mathrm{AU}$, respectively. The exact density scale is not given because the rendering technique would not preserve it well (generally, high density regions are indicated by yellow and red, and low density regions are indicated by green and blue).

compatible with the values used in our simulations. A comparison of our models with real symbiotic systems is therefore well-founded.
There are two distinct classes of symbiotic stars: the S-type with normal giants and orbital periods of order a few years, and the D-type with Mira primaries for which 


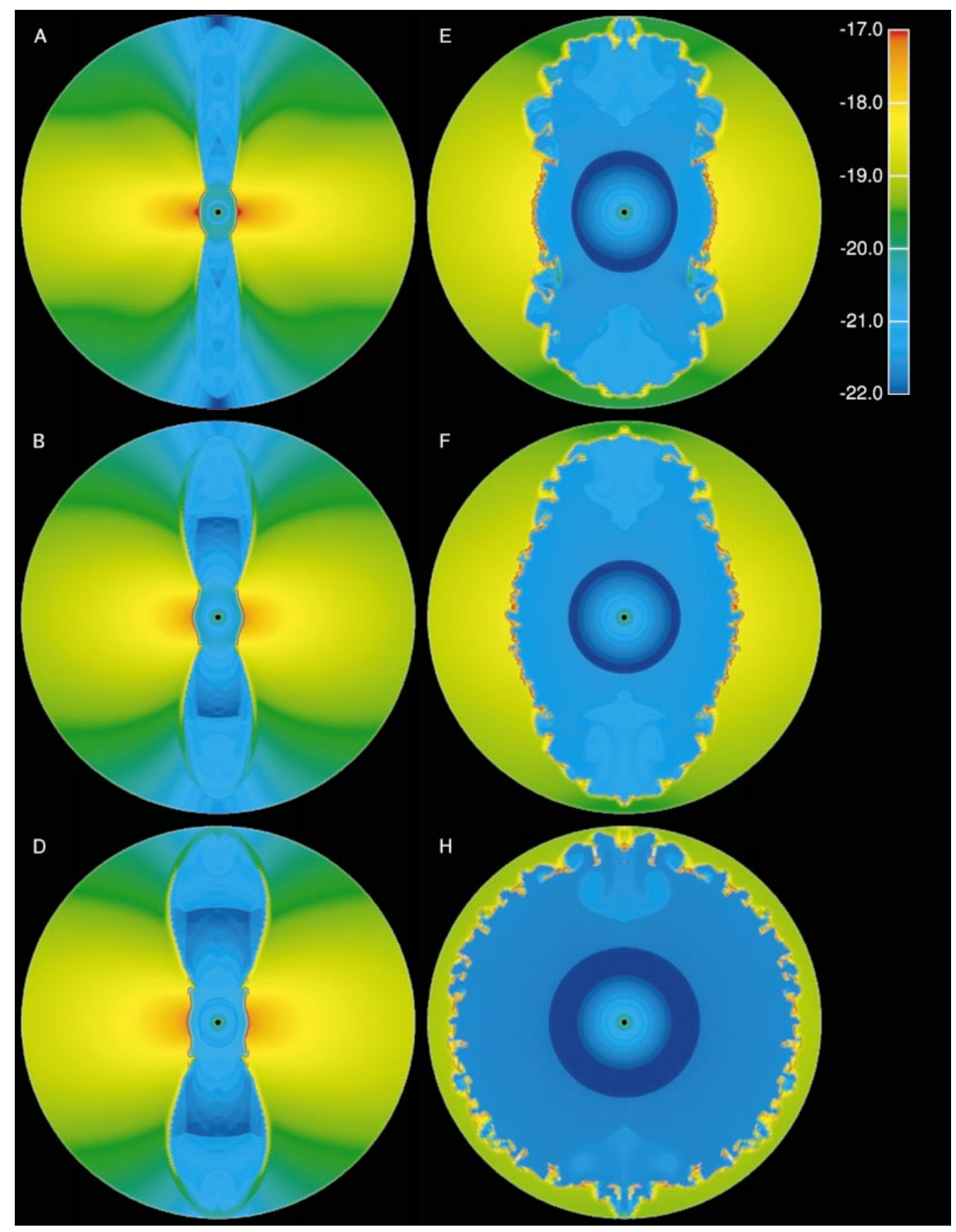

Fig. 6. Density distributions in models A, B, D, E, F, and H. The snapshots are taken at the same times as in Fig. 5. The scale on the bar shows logarithm of density in $\mathrm{g} \mathrm{cm}^{-3}$.

the orbital periods are generally not known, presumably because they are too long (>50yr; Mikołajewska 2001) to be measured. Our study indicates that the slow wind can be significantly modified by the secondary component's gravity only in close binaries with the orbital separations smaller than $10 \mathrm{AU}$ (models A-G), i.e. only in the S-type symbiotic systems, whereas the much wider D-type systems should not show significant asymmetry.

However, optical and radio imaging of symbiotic stars reveal a picture which is much more complicated than that. Extended ionized nebulae are associated with both S-type systems ( 5 cases) and symbiotic Miras ( 8 cases). In all 13 cases the nebulae are aspherical, often with bipolar lobes and jet-like components (Corradi et al. 1999). Observations also suggest that the nebulae are elongated perpendicularly to the orbital plane except in some cases, where the bipolar outflow is likely to be inclined at less than $90^{\circ}$ to the orbital plane (Mikołajewska 1999, and references therein). This may be however an observational effect because images of ionized nebulae show regions 
with some specific electron density and temperature which may be different for various emission lines and radio frequencies.

There is also an additional, indirect evidence from emission line profiles that in many symbiotic systems the distribution of the dense wind is aspherical. For example, $\mathrm{H} \alpha$ profiles with a strong reversal slightly blueshifted from the line center are observed in many cases together with strong double-peaked [O III] emission lines (e.g. Ivison et al. 1994). The $\mathrm{H} \alpha$ absorption is especially remarkable in systems with high orbital inclination, and it is usually stationary with respect to the orbital motion, which supports the idea that it arises from self-absorption in a neutral portion of the dense wind. In some cases the absorption remains strong over the whole orbital cycle, indicating that the ionized nebula is probably bounded on all sides by significant amount of neutral material, at least near the orbital plane. On the other hand, the presence of strong forbidden lines requires an extended low-density ionized region. This suggests that those nebulae have a bipolar ionization structure, with much lower density at the poles than in the orbital plane.

Generally, observational evidence based on symbiotic stars suggests that bipolarity of nebulae is indeed related to binarity of their central stars. It is clear, however, that the gravitational focusing cannot explain all features observed in those objects. In particular, optical and radio imaging show extended bipolar nebulae in D-type systems (e.g. Corradi et al. 2000, and references therein) whereas our models indicate that focusing is is inefficient in those cases. Thus, their bipolar structures must originate either from intrinsic asphericity of the dense wind (or both winds), or from effects associated with the presence of an accretion disk around the hot component. The symbiotic Miras in D-systems are extreme in the sense that their pulsation periods are systematically longer, and their mass loss rates are systematically higher than the typical pulsation periods and mass loss rates for single galactic Miras. An intrinsic asymmetry in the AGB winds from symbiotic Miras might account for the shapes of their nebulae, but its origin has yet to be found. It is conceivable that interaction of the secondary with a pulsating primary could affect the mass outflow from the latter, but both theory and observational evidence are missing.

HST and ground-based images reveal a complex ionized nebula around the symbiotic system $\mathrm{CH}$ Cyg, extending up to about $5000 \mathrm{AU}$ from the central stars (Corradi et al. 2001). The orbital parameters of $\mathrm{CH}$ Cyg, $P_{\text {orb }} \sim$ $15 \mathrm{yr}$ and $a \sim 7 \div 8 \mathrm{AU}$ (e.g. Belczyński et al. 2000) are similar to parameters of our models E-F. It is interesting that in this case the bipolar structure of the nebula is hidden in the dominating elliptical shape of the main body. This is consistent with our finding that the transition between bipolar and elliptical morphology should happen in systems resembling Model E.

Our close binary models A, B and C, can account for the extended nebulosity in the symbiotic nova V1329 Cyg $\left(P_{\text {orb }}=956.5\right.$ days $)$. The nebula - detected in
[O III] $\lambda 5007 \AA$ emission line - is aligned with the orbital plane, and its size exceeds the binary separation by over 2 orders of magnitude (Schild \& Schmid 1997). In 1964, the hot component of V1329 Cyg underwent strong nova eruption, and for many years following the outburst it was a copious source of high-energy photons $\left(\gtrsim 10^{48}\right.$ phot $^{-1}$; e.g. Mürset \& Nussbaumer 1994). The symbiotic nova eruption is usually followed by a phase of intense fast wind from the hot component which efficiently depletes material above and below the orbital plane, leaving only the densest portion in place. It is possible that the observed nebula is the ionized part of that portion, roughly corresponding to the inner edge of the red, disk-like region in Figs. 5 and 6.

\section{Conclusions}

We have studied the effect of gravitational focusing on the morphology of PNe with detached binary cores. The gravity of the secondary influences the nebula indirectly, by modifying the density and velocity distribution of the AGB wind from the primary. A fast, low-density postAGB wind expanding in such an environment produces planetary nebulae with shapes ranging from spherical to very elongated bipolar. Among various mechanisms invoked to explain the origin of bipolar nebulae (see e.g. Soker 1998; Corradi \& Schwarz 1995; Reimers et al. 2000; García-Segura et al. 1999; Matt et al. 2000; Mastrodemos \& Morris 1998, 1999) this one is particularly simple, and free of arbitrary assumptions: a bipolar nebula is created whenever an AGB star is a member of a close binary. Magnetized or rotating stellar winds are redundant (obviously they are still needed in wide binaries or when the AGB star is single or when more exotic morphologies have to be accounted for).

We believe that our models successfully recover the basic workings of the gravitational focusing mechanism. However, some details could still be clarified. We have neglected all radiative effects. As a result, a hot "halo" is formed around the secondary, and the flow of the AGB wind toward the orbital plane is partly inhibited. Luckily, this means that we underestimate the efficiency of our mechanism, i.e. the basic conclusions of our work are not affected. However, with cooling included, one might hope to put tighter limits on binary parameters at which bipolar nebulae would most likely emerge. Entirely realistic models would also have to include a detailed description of wind acceleration, and the effects of radiation emitted by the secondary (especially in the case of symbiotic stars, where the secondary is able to ionize the AGB wind).

Separate problems arise from limitations of numerical techniques and approximations. Our SPH simulations of AGB winds are fully three-dimensional. We have argued that the amplitude of any nonaxisymmetric effects would decrease with increasing distance from the binary, so that an averaging procedure may be applied to the models, yielding axisymmetric configurations. The averaging procedure does not affect global morphology of the nebula, 
however finer structural details are lost. Such details might be produced, for example, by spiral features appearing in SPH models due to the orbital motion of the secondary. It is conceivable that the spirals would leave an imprint on the shell separating the AGB wind from the fast wind. The resulting nebula might have a structure similar to that observed e.g. in NGC 6543 or NGC 7293. However, extensive 3-D simulations are necessary to check this possibility.

Our calculations show that in the majority of, and perhaps in all, S-type symbiotic systems the dense wind from the giant primary must be strongly focused towards the orbital plane due to gravitational interaction with the secondary, and such a picture is supported by observations. This means that all models of those systems based on the assumption of a spherically symmetric dense wind, such as the STB (Seaquist et al. 1984) model for radio emission, should be revised. We have also demonstrated that the gravitational interaction with the secondary is not sufficient to account for all observed morphologies of nebulae associated with symbiotic Miras (however, the possibility that their binarity can give rise to significant intrinsic asymmetry of their winds cannot be ruled out).

Acknowledgements. This research was supported by the Committee for Scientific Research through grants 2P03D 01419 and 5P03D 019 20. The original version of the SPH code was kindly provided by Dr. Gregory Laughlin. We also thank Adam Frankowski for fruitful discussions.

\section{Appendix A: Free wind solution}

In a spherically-symmetric stationary wind $\rho, v$ and $p$ are functions of $r$ only. Continuity equation reduces to

$\rho=\frac{\alpha}{r^{2} v}$

(where $\alpha=\dot{M} / 4 \pi$ ), and momentum equation - to

$v \frac{\mathrm{d} v}{\mathrm{~d} r}=-\frac{1}{\rho} \frac{\mathrm{d} p}{\mathrm{~d} r}$.

Eliminating $p$ with the help of polytropic relation we get

$v \frac{\mathrm{d} v}{\mathrm{~d} r}=\kappa \gamma \rho^{\gamma-2} \frac{\mathrm{d} \rho}{\mathrm{d} r}$.

Differentiation of (A.1) yields an additional relation between derivatives of $\rho$ and $v$ which, combined with (A.3), gives

$\frac{\mathrm{d} \rho}{\mathrm{d} r}=\frac{\rho}{r} \frac{2}{\kappa \alpha^{-2} \gamma r^{4} \rho^{\gamma+1}-1}$

or a similar expression for $\frac{\mathrm{d} v}{\mathrm{~d} r}$.

\section{References}

Belczyński, K., Mikołajewska, J., Munari, U., Ivison, R. J., \& Friedjung, M. 2000, A\&AS, 146, 407

Bond, H. E. 2000, in ASP Conf. Ser. 199, Asymmetrical Planetary Nebulae II, From Origins to Microstructures, 115

Corradi, R. L. M. 2000, in ASP Conf. Ser. 199, Asymmetrical Planetary Nebulae II, From Origins to Microstructures, 25

Corradi, R. L. M., Brandi, E., Ferrer, O. E., \& Schwarz, H. E. 1999, A\&A, 343, 841

Corradi, R. L. M., Livio, M., Schwarz, H. E., \& Munari, U. 2000, in ASP Conf. Ser. 199, Asymmetrical Planetary Nebulae II, From Origins to Microstructures, 175

Corradi, R. L. M., Munari, U., Livio, M., et al. 2001, ApJ, 560, 912

Corradi, R. L. M., \& Schwarz, H. E. 1995, A\&A, 293, 871

Dorfi, E. A., \& Höfner, S. 1996, A\&A, 313, 605

Dwarkadas, V. V., \& Balick, B. 1998, ApJ, 497, 267

Frankowski, A., \& Tylenda, R. 2001, A\&A, 367, 513

García-Segura, G., Langer, N., \& Różyczka, M. 1999, ApJ, 517, 767

Hernquist, L., \& Katz, N. 1989, ApJS, 70, 419

Ivison, R. J., Bode, M. F., \& Meaburn, J. 1994, A\&AS, 103, 201

MacDonald, J., \& Bailey, M. E. 1981, MNRAS, 197, 995

Mastrodemos, N., \& Morris, M. 1998, ApJ, 497, 303

Mastrodemos, N., \& Morris, M. 1999, ApJ, 523, 357

Matt, S., Balick, B., Winglee, R., \& Goodson, A. 2000, ApJ, 545,965

Mellema, G. 1995, MNRAS, 277, 173

Mellema, G. 1997, A\&A, 321, L29

Mellema, G., \& Frank, A. 1995, MNRAS, 273, 401

Mikołajewska, J. 1999, in ASP Conf. Ser. 188, Optical and Infrared Spectroscopy of Circumstellar Matter, 291

Mikołajewska, J. 2000, in ASP Conf. Ser. 199, Asymmetrical Planetary Nebulae II, From Origins to Microstructures, 431

Mikołajewska, J. 2001, in ASP Conf. Ser. 246, Small-Telescope Astronomy on Global Scales, ed. W. C. B. Paczyński, \& C. Lemme, 167

Mikołajewska, J., Ivison, R. J., \& Omont, A. 2001, Adv. Space Res., in press

Monaghan, J. J., \& Lattanzio, J. C. 1985, A\&A, 149, 135

Mürset, U., \& Nussbaumer, H. 1994, A\&A, 282, 586

Reimers, C., Dorfi, E. A., \& Höfner, S. 2000, A\&A, 354, 573

Schild, H., \& Schmid, H. M. 1997, A\&A, 324, 606

Seaquist, E. R., Taylor, A. R., \& Button, S. 1984, ApJ, 284, 202

Soker, N. 1998, ApJ, 496, 833

Stone, J. M., \& Norman, M. L. 1992, ApJS, 80, 753

Walder, R., \& Folini, D. 1998, A\&A, 330, L21

Winters, J. M., Le Bertre, T., Jeong, K. S., Helling, C., \& Sedlmayr, E. 2000, A\&A, 361, 641 